\title{
Value of oesophagoscopy and bronchoscopy in diagnosis of synchronous malignancies in patients with head and neck squamous cell carcinomas: a 10-year single center experience in Chinese population.
}

\author{
Shi Yeung $\mathrm{Ho}^{1}$ and Raymond Tsang ${ }^{2}$ \\ ${ }^{1}$ Queen Mary Hospital \\ ${ }^{2}$ University of Hong Kong
}

May 19, 2020

\begin{abstract}
Title Value of oesophagoscopy and bronchoscopy in diagnosis of synchronous malignancies in patients with head and neck squamous cell carcinomas: a 10-year single center experience in Chinese population. Objectives Routine screening of patients with head and neck squamous cell carcinomas (HNSCCs) for synchronous malignancies using oesophagoscopy and bronchoscopy had been controversial. The aim of this study is therefore to find out the rate of synchronous malignancies in patients with primary HNSCCs, the risk factors for its occurrence and the effectiveness of oesophagoscopy and bronchoscopy in a single center with Chinese population. Method A retrospective review of medical records was conducted from July 2008 to June 2018 in a tertiary referral center in Hong Kong. All patients with newly diagnosed HNSCCs were screened with oesophagoscopy and bronchoscopy at the time of diagnosis and therefore all patients were included in the study. The incidence of synchronous malignancies along the aerodigestive tract and the yield of oesophagoscopy and bronchoscopy were studied. Result Of the 702 patients included in the study, the overall rate of synchronous malignancies was $8.3 \%(58 / 702)$, with the rate of synchronous esophageal and lung malignancies being $5.8 \%(41 / 702)$ and $0.85 \%$ (6/702) respectively. 14 out of the 41 esophageal malignancies were only detectable with esophagoscopy. Only one of the synchronous lung malignancies was detectable by bronchoscopy. Risk factors for synchronous malignancies include male gender, smokers, drinkers and primary hypopharyngeal cancer. Conclusion Oesophagoscopy is essential for detecting synchronous esophageal malignancies in patients with HNSCC especially in male patients, smokers, drinkers and primary hypopharyngeal cancer patients. Bronchoscopy had a low yield for synchronous lung malignancies and can be potentially replaced by imaging techniques.
\end{abstract}

\section{Introduction}

Synchronous malignancies occurring along the upper aerodigestive tract was originally discovered by Billroth ${ }^{1}$ and Slaughter ${ }^{2}$, with the latter proposing the theory of 'field cancerization'. The theory of 'field cancerization' suggested that with exposure to tobacco and alcohol, synchronous multifocal malignancies can occur in oesophagus and lung for patients with primary head and neck squamous cell carcinomas (HNSCCs). The rate of occurrence of such synchronousmalignancies can be as high as $23 \%^{3-6}$ and their overall survival can be $50 \%$ worse when compared with first primaries. Oesophagoscopy and bronchoscopy had beenthe procedure of choice for screening synchronous malignancies along the upper aerodigestive tract ${ }^{7-9}$. Debates however existed on whether the routine use of them were warranted as there were concerns over risks and cost of procedure and increasing waiting time before start of treatment ${ }^{10-13}$. The aim of this study is therefore to find out the rate of such synchronousmalignancies in patients with primary HNSCCs, the risk factors for its occurrence and the effectiveness of oesophagoscopy and bronchoscopy in a single center with Chinese population. 


\section{Method}

A retrospective review of electronic medical records was conducted on consecutive patients with newly diagnosed and untreated HNSCCsin a tertiary referral center. In our center, newly diagnosed HNSCC patients were screened with oesophagoscopy and bronchoscopy at the time of diagnosisand therefore all patients were included in the study.The endoscopic procedure was done in outpatient setting under local anesthesia and using trans-nasal flexible endoscopy. With the trans-nasal flexible endoscopy, the nasopharyngeal, oropharyngeal, laryngeal, and pharyngeal areas will be inspected, following by oesophagoscopy (for oesophagus down to oesophageal gastric junction) and bronchoscopy (for tracheobronchial tract).

Synchronousmalignancies were defined as malignancies that were detected during the initial workup upon the diagnosis of the primary malignancy at other sites in the upper aerodigestive tract.Exclusion criteria included HNSCC arising fromnon-aerodigestive tract region in head and neck region (ear, skin, salivary glands, lacrimal glands), tumorswith non-squamous cell carcinomas histology, patient with history of nasopharyngeal carcinomas, patients with previous history of irradiation and patients of non-East Asian ethnicity. Information regarding patient's demographics, subsites and staging of primary and synchronousmalignancies, pickup rate by other means of investigations like PET-CT (Positron emission tomography- computed tomography scan), survival statistics (disease free survival and overall survival) will be recorded and studied. Staging of primary and synchronousmalignancies was done in accordance to the eighth edition of the AJCC Cancer Staging Manual (TNM classification from the American Joint Committee on Cancer [AJCC]).

All data was input into Excel spreadsheets (Microsoft Excel for Mac 2013 version 15; Microsoft Corporation, Redmond, Washington). Data analysis was done with SPSS version 24.0 software (IBM, Armonk, New York). Differences in means for normally distributed variables was assessed with 2-tailed unpaired t test. Differences in means for non-normally distributed variables was assessed with Mann-Whitney U test. P value was set at $<0.05$ to be statistically significant.

\section{Results}

Table 1 summarizes the characteristicsof the cohort of patients. There were 702 consecutive patients with newly diagnosed and untreated HNSCCs with oesophagoscopy and bronchoscopy done at primary diagnosis in the inclusion period. There were 522 male and 180 female patients. The mean age of the patients was 63 years old (range,25-94 years old). There were 393 patients (56\%)who were active or ex-smokers, 209 patients (29.8\%)who were drinkers or ex-drinkers and 182 patients $(25.9 \%)$ who were smokers and drinkers.

Majority of sites of primary malignancies were in the oral cavity (356 patients, $50.7 \%$ ), followed by laryngeal (126 patients, 17.9\%), hypopharyngeal (110 patients, 15.7\%), oropharyngeal (74 patients, 10.5\%) and paranasalsinuses and nasal cavity cancers (33 patients, $4.7 \%$ ). For staging, most of the patients were having stage 4 disease on presentation (357 patients, 50.9\%), whereas there were $162(23.2 \%), 92(13.1 \%)$ and 89 (12.7\%) patients with stage 1,2 and 3 disease respectively.

The rate of synchronousmalignancies was $8.3 \%$ (58/702 patients).Figure 1 and 2 shows the Kaplan-Meier survival curve showing overall survival and disease-free survival of the cohort. When comparing between patients with synchronousmalignancies and patients with no synchronousmalignancies (single primary malignancy), the one-year and three-year overall survival (OS) rate were significantly lower in the former group than the latter (one-year OS $40.8 \%$ vs $83.25 \%$, P $<0.0005$; three-year OS $6.8 \%$ vs $54.0 \%, \mathrm{P}<0.0005$ ). The same would apply for disease free survival (DFS) rate (one-year DFS $24.5 \%$ vs $68.8 \%, \mathrm{P}<0.0005$; three-year DFS $6.8 \%$ vs $50.1 \%, \mathrm{P}<0.0005)$.

Characteristics of synchronousmalignancies were delineated in table 2. Among all primary subsites, hypopharynx carried the highest rate of synchronousmalignancies at $27.3 \%$ (30/110 patients). There was a relatively low incidence of synchronousmalignancies in other primary subsites. In terms of sites of synchronousmalignancies, most of them occurred in oesophagus (41/58 patients, $73.2 \%$ ), followed by sites other than the aerodigestive tract $(9 / 58$ patients, $15.5 \%)$, lung (5/58 patients, $8.9 \%)$, head and neck $(3 / 58$ patients, $5.4 \%)$. 
There were three synchronousmalignancies in the head and neck region.Amongst them, two were detectable on clinical examination (one at tongue from a primary hypopharyngeal cancer and another at lower alveolus from a primary cancer at maxillary sinus). The remaining one was at tongue base from a primary retromolar trigone cancer. It was non-suspicious at initial endoscopy but showed increase uptake when working up with PET-CT and therefore biopsy was performed to confirm the malignancy.

The rate of synchronousoesophagealmalignancies was $5.8 \%$ (41/702 patients). The majority of the synchronousoesophagealmalignancies were suffering from primary hypopharyngeal cancers $(25 / 41$ patients, $61.0 \%)$, following by oral cavity cancers (8/41 patients, $19.5 \%)$, laryngeal cancers (4/41 patients, $9.8 \%)$ and oropharyngeal cancers (4/41 patients, $9.8 \%$ ). Most of the synchronousoesophagealmalignancies detected were early diseases, with $53.7 \%$ of them having stage 1 disease. Risk factors for synchronousoesophagealmalignancies included male gender (Odds ratio OR 14.89, 95\% CI: 2.03-109.08), smoker status (OR 3.33, 95\% CI: $1.51-7.30$ ), drinker status (OR 26.14, 95\% CI: $9.18-74.40$ ) and primary hypopharyngeal cancer (OR 10.59, 95\% CI: $5.43-20.64$ ). Out of the 41 patients with synchronousoesophagealmalignancies, 14 of them (34\%) wereonly detectable with oesophagoscopy and not by other means of investigation including $\mathrm{CT}$ scans of the thorax and PET-CT scans. Therefore, the exclusive detection rate for oesophagoscopy among all subjects was $2.0 \%$ (14/702 patients). 4 of these 14 patients were asymptomatic., 13 of themwere having stage 1 diseaseand only 1 of them was having a stage 3 disease. The overall survival and disease-free survivalof patients with oesophagealmalignancies exclusively detectable with oesophagoscopy were significantly better when comparing with other synchronousoesophagealmalignancies not exclusively detected by oesophagoscopy $(\mathrm{P}=0.028)$.

The rate of synchronous lung malignancies was $0.7 \%$ (6/702 patients). Among these 6 patients, 4 of themwere detected on chest x-ray, one by PET-CT and one by bronchoscopy. However, all of themwere detectable by PET-CT when being further worked up. Therefore, the exclusive detection rate by bronchoscopy is $0 \%$.

No complications oesophageal or bronchial perforation occurred amongst all patients in the study period. The medianfollow-up period for the cohort of patients was 23 months (Range: 0-120 months).

\section{Discussion}

The role of oesophagoscopy in HNSCC had been debatable in the literature. Amongst North American studies, the rate of synchronous malignancies in oesophagus ranged from 0-8\% in most studies in the 1980s. However, in a review by Mcgarey et $\mathrm{al}^{14}$, there had not been a single synchronous oesophagealmalignancy detected on staging oesophagoscopy for patients with HNSCC since 2000 in North American studies. In contrast, most studies fromTaiwan and Hong Kong however had found significant rates of synchronous oesophagealmalignancies among patients with HNSCCs. Chow et al ${ }^{15}$ reported a rate of $10 \%$ for clinically important oesophageal lesions found among 118 HNSCC patients undergoing oesophagoscopy for workup. Chung et $\mathrm{al}^{6}$ and Huang et $\mathrm{al}^{16}$ reported $23.3 \%$ and $14.8 \%$ synchronous oesophagealmalignancies among patients with HNSCCs respectively in their studies. These findings could be explained by the relatively higher prevalence rate of oesophagealmalignancies in $\mathrm{Asia}^{17}$ and the high prevalence of habits of chewing betel nuts in Taiwan ${ }^{16,18}$, which is a common carcinogen for both oesophagealmalignancies and HNSCCs.

In our study, the rate of occurrence of synchronousoesophagealmalignancies was $5.8 \%(41 / 702)$ and out of these 41 lesions, 14 of them were onlydetectable by oesophagoscopy, i.e. these malignancies would be otherwise missed by other means of investigation (e.g. CT/PET-CT). 4 of these 14 patients were asymptomatic. In addition, most of these malignancies (13/14) found were of early stage (stage 1 cancers). The overall survival and disease-free survival of patients with malignancies exclusively detectable with oesophagoscopy were significantly better when comparing with other synchronousoesophagealmalignancies not exclusively detectable with oesophagoscopy $(\mathrm{P}=0.028)$. This had highlighted the importance of staging oesophagoscopy in screening out synchronous oesophagealmalignancies. It is also effective in screening out early stage disease and potentially benefit patient's survival.

Inour study, the risk factors associated with development of synchronousoesophagealmalignancies included male gender, smoker/drinker status and primary hypopharyngeal cancer.Anatomically, the hypopharynx 
and oesophagus are structures connected continuously and there had been proven association between malignancies from both structures ${ }^{16,18-21}$. Huang et $\mathrm{al}^{16}$ evaluated 248 patients with hypopharyngeal cancers prospectively and reported a rate of $14.8 \%$ of synchronous oesophageal cancers and $9.4 \%$ of oesophageal dysplasia among the patients. The French ENT society concluded in their 2012 guideline that oesophagoscopy was indicated as staging procedure for patients with hypopharyngeal cancers ${ }^{22}$.

As the field cancerization theory ${ }^{2}$ suggested, alcohol consumption and tobacco use are risk factors for development of synchronous malignancies in the upper aerodigestive tract. Lower rates of synchronous malignancy development among non-smokers or non-drinkers was found in studies ${ }^{23,24}$. Alcohol drinking was reported to be associated with increased risk of oesophagealmalignancy in a study for patients with $\mathrm{HNSCCs}^{6}$ (OR:5.90, $\mathrm{P}=0.020$ ), as well as an independent risk factor for development of oesophagealcancerous or dysplastic lesions in hypopharyngeal cancer patients ${ }^{16}$ (OR: 6.95, $\left.\mathrm{P}<0.05\right)$. The French ENT society suggested that oesophagoscopy should be indicated for patients with chronic alcohol intoxication as it increased the risk of development of synchronous oesophagealmalignancies ${ }^{22}$.

Bronchoscopy had been less utilized as a routine screening procedure for synchronous lung malignancies in patients with HNSCC when comparing with oesophagoscopy. This could be due to the availability of other non-invasive means of screening modalities such as chest x-ray, CT scan of the thorax and PET-CT imaging. There was also a low yield of bronchoscopy in the literature as a screening modality for HNSCC patients, ranging from $0-1 \%^{21}$. In our study, the rate of synchronouslung malignancies was at $0.6 \%$, comparable with the literature. Amongst them, non-of them is exclusively detectable with bronchoscopy and all of them can be detectable on PET-CT, suggesting that bronchoscopy can be potentially replaced by other investigative modalities.

\section{Conclusion}

Oesophagoscopy is recommendedfor screening synchronousoesophagealmalignancies in patients with HNSCCs, in particular for patients who were smokers or drinkers, male patients and hypopharyngeal cancer patients. Part of these synchronousoesophagealmalignancies are also only detectable with oesophagoscopy and can potentially affect the survival of patients. Due to the low yield of bronchoscopyand can be potentially replaced by other means of investigation, it is not recommended as a screening procedure for patients with HNSCCs. Complication rate of oesophagoscopy and bronchoscopy is extremely low and the procedure is well tolerated using trans-nasal flexible endoscopy.

\section{Keypoints}

- The concept of 'field cancerization' applies to patients with head and neck squamous cell carcinoma

- Synchronous, multifocal malignancies can occur along the upper aerodigestive tractwith common exposure to tobacco and alcohol

- Oesophagoscopy is recommended for screening synchronous oesophagealmalignancies in particular for patients who are smokers or drinkers, male patients and hypopharyngeal cancer patients

- Bronchoscopy is not recommended for screening synchronous lung malignancies

- Endoscopy using trans-nasal flexible endoscopic technique is well tolerated with extremely low complication rate

\section{Table 1.}

Patient characteristics, sites and staging of primary malignancies

Parameter

Mean Age at presentation (years \pm SD)

Gender (n, \%) Male Female

Active or ex-smokers (n,\%) Yes No

Active or ex-drinkers (n, \%) Yes No

Sites of primary malignancies (n, \%) Oral cavity Larynx Hypopharynx Oropharynx Paranasal sinuses and nasal cavity
$\mathrm{Nl}$ 
Parameter $\mathrm{Nl}$

Stages of primary malignancies (n, \%) Stage 1 Stage 2 Stage 3 Stage 4

16

Table 2.

Characteristics of synchronousmalignancies and synchronousoesophagealmalignancies

\section{Parameters}

Sites of synchronousmalignancies (n, \% among all synchronousmalignancies) Oesophagus Lung Head and neck Other sites * Sites of primary malignancies giving rise to synchronousmalignancies (n, $\%$ among total number of patients with the primar Rate of synchronousoesophagealmalignancies (n, \%)

Sites of primary malignancies giving rise to synchronousoesophagealmalignancies(n, \% among total number of patients with Stages of synchronousprimary oesophagealmalignancies(n, \%) Stage 1 Stage 2 Stage 3 Stage 4

Risk factors for synchronousoesophagealmalignancies(Odds ratio) Male gender Smoker status Drinker status Primary hypop

*Other sites (number): prostate (4), liver (2), sigmoid (1), thyroid (1), stomach (1)

\section{Figure 1.}

Kaplan-Meier survival curve. Comparing overall survival of patients with synchronousmalignancies and single primary malignancies.

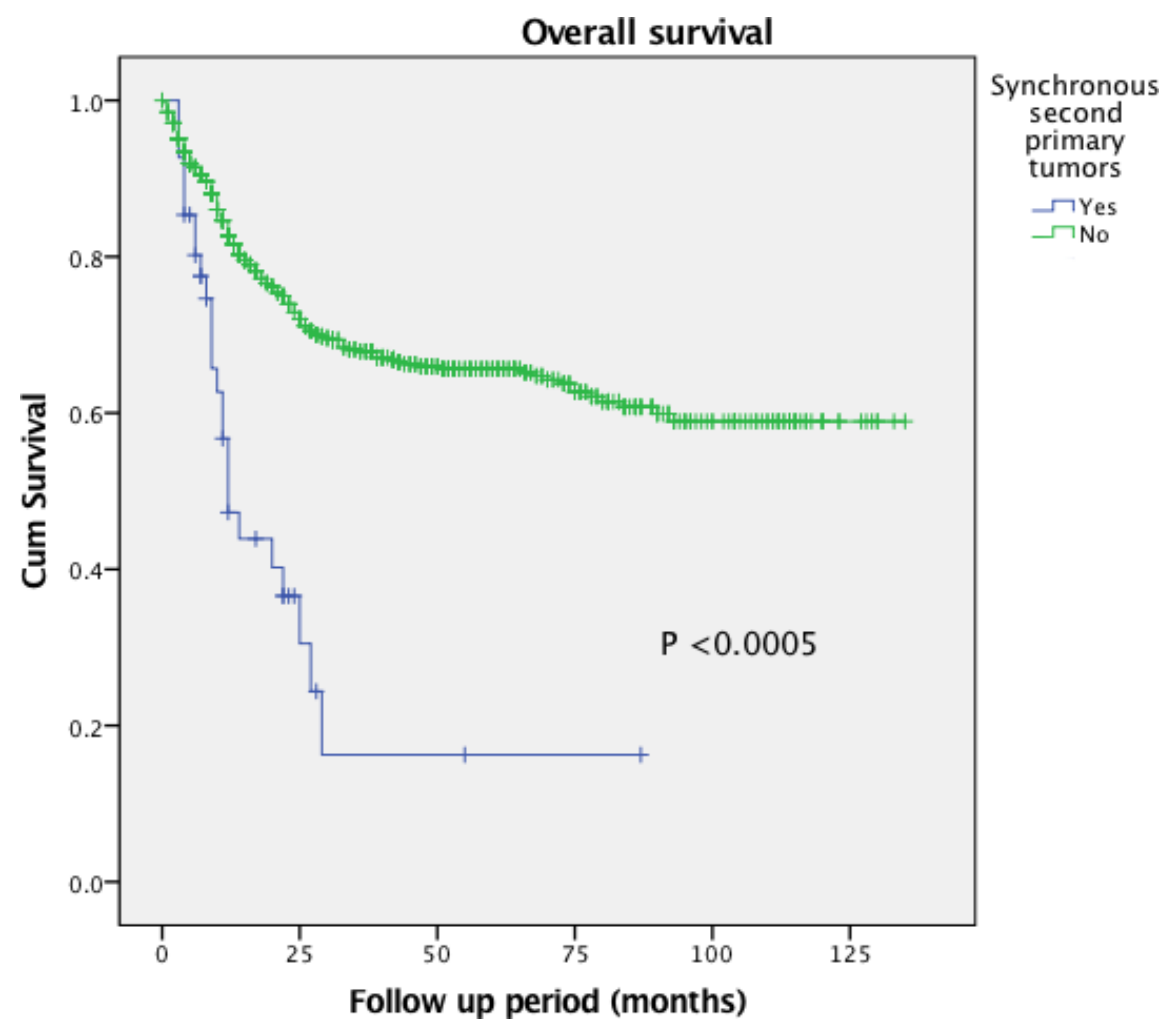

Figure 2. 
Kaplan-Meier survival curve. Comparing disease-free survival of patients with synchronousmalignancies and single primary malignancies.

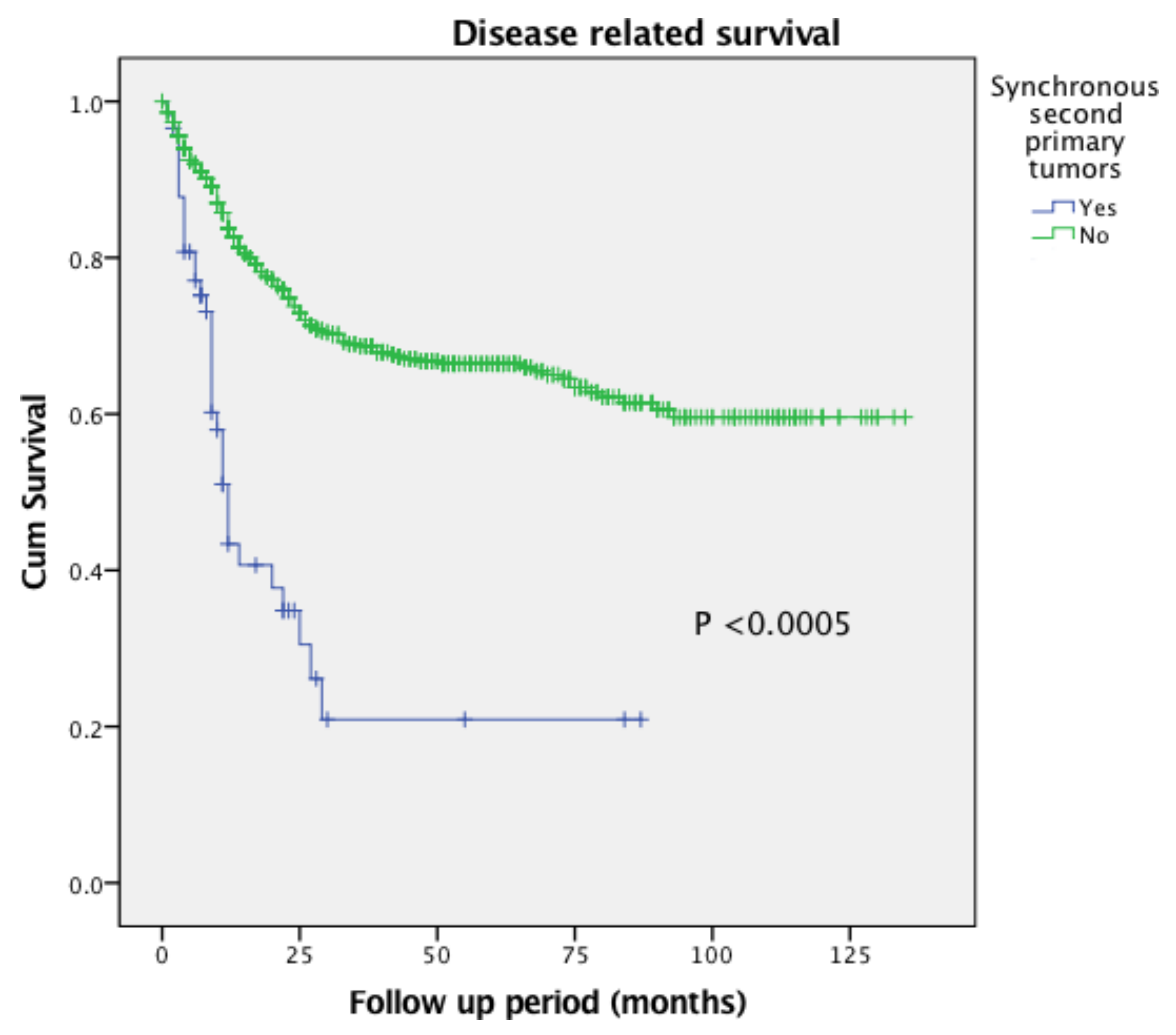

\section{References}

1. Billroth T, Von Winiwarter A. A general surgical pathology and therapeutics. New York: Appleton; 1883.

2. Slaughter DP, Southwick HW Smejkal W. Field cancerization in oral stratified squamous epithelium; clinical implications of multicentric origin. Cancer 1953: 963-968.

3. Bhattacharyya N, Nayak JK. Survival outcomes for second primary head and neck cancer: a matched analysis. Otolaryngol Head Neck Surg 2005;132:63-68

4. Schwarz LH, Ozsahin M, Zhang GN, et al. Synchronous and metachronous head and neck carcinoma. Cancer 1994;74:1933- 1938

5. Di Martino E, Sellhaus B, Hausmann R, et al. Survival in sec-ond primary malignancies of patients with head and neck cancer. J LaryngolOtol2002;116:831-838.

6. Chung CS,LiaoLJ,LoWC,etal.Riskfactorsfor second primary neoplasia of oesophagus in newly diagnosed head and neck cancer patients. BMC Gastroenterol. 2013;13:154.

7. McGuirt WF. Panendoscopy as a screening examination for synchronous primary cancers in head and neck cancer: a prospective sequential study and review of the literature. Laryngoscope 1982;92: $569-576$.

8. Maisel RH, Vermeersch H. Panendoscopy for second primaries in head and neck cancer. Ann OtolRhinolLaryngol1981;90: 460-464.

9. Abemayor E, Moore DM, Hanson DG. Identification of synchronous oesophagealcancers in patients with head and neck cancer. J Surg Oncol 1988;38: 94- 96.

10. Colt HG, Matsuo T. Hospital charges attributable to bronchoscopy-related complications in outpatients. Respiration 2001;68:67-72 
11. Ginzburg L, Greenwald D, Cohen J/ Complications of endoscopy. GastrointestEndosc Clin N Am 2007;17:405-432

12. Soni A, Sampliner RE, Sonnenberg A. Screening for high-grade dysplasia in gastrooesophageal reflux disease: is it cost- effective? Am J Gastroenterol 2000;95:2086-2093

13. Gupta N, Bansal A, Wani SB, Gaddam S, Rastogi A, Sharma P. Endoscopy for upper GI cancer screening in the general population: a cost-utility analysis. GastrointestEndosc2011;74:610-624

14. McGarey PO, O'Rourke AK, Owen SR, et al. Rigid Oesophagoscopy for Head and Neck Cancer Staging and the Incidence of Synchronous Oesophageal Malignant Neoplasms. JAMA Otolaryngol Head Neck Surg. 2016;142(1):40-45.

15. Chow TL, Lee DT, Choi CY, Chan TT, Lam SH. Prediction of synchronousoesophageal lesions in head and neck squamous cell carcinoma: a multivariate analysis. Arch Otolaryngol Head Neck Surg 2009;135:882-885

16. Huang YC, Lee YC, Tseng PH, et al. Regular screening of oesophageal cancer for 248 newly diagnosed hypopharyngeal squamous cell carcinoma by unsedatedtransnasal esophagogastroduodenoscopy. Oral Oncol 2016;55: 55-60.

17. Zhang H-Z, Jin G-F, Shen H-B. Epidemiologic differences in oesophageal cancer between Asian and Western populations. Chinese Journal of Cancer. 2012;31(6):281-286.

18. Hung SH, Tsai MC, Liu TC, Lin HC, Chung SD. Routine endoscopy for oesophageal cancer is suggestive for patients with oral, oropharyngeal and hypopharyngeal cancer. PLoS ONE 2013;8.

19. Chuang SC, et al. Risk of second primary cancer among patients with head and neck cancers: a pooled analysis of 13cancer registries. Int J Cancer 2008;123(10):2390 - 6.

20. Stoeckli SJ, Zimmermann R, Schmid S. Role of routine panendoscopy in cancer of the upper aerodigestive tract. Otolaryngol Head Neck Surg 2001;124:208-12

21. GuardiolaE,ChaigneauL,VillanuevaC,PivotX.Istherestillarolefortripleendoscopy as part of staging for head and neck cancer? CurrOpinOtolaryngol Head Neck Surg. 2006;14(2):85-88.

22. de Mones E, Bertolus C, Salaun PY, Dubrulle F, Ferrie JC, Temam S, Chevalier D, Vergez S, Lagarde F, Schultz P, Lapeyre M, Barry B, Tronche S, de Raucourt D, Moriniere S. Initial staging of squamous cell carcinoma of the oral cavity, larynx and pharynx (excluding nasopharynx). Part 2: remote extension assessment and exploration for secondary synchronous locations outside of the upper aerodigestive tract. 2012 SFORL guidelines. Eur Ann Otorhinolaryngol Head Neck Dis 2013;130:107-112

23. Koo K, Harris R, Wiesenfeld D, Iseli TA. A role for panendoscopy? Second primary tumour in early stage squamous cell carcinoma of the oral tongue. The Journal of Laryngology \& Otology. 2015;129(S1):S27-S31.

24. Rodriguez-Bruno, K., Ali, M. J. and Wang, S. J. Role of panendoscopy to identify synchronous second primary malignancies in patients with oral cavity and oropharyngeal squamous cell carcinoma. Head Neck, 2011;33: 949-953.

25. Boiselle PM. Computed tomography screening for lung cancer. JAMA 2013;309:1163-1170

\section{Hosted file}

Figure 1.docx available at https://authorea.com/users/323818/articles/452304-value-ofoesophagoscopy-and-bronchoscopy-in-diagnosis-of-synchronous-malignancies-in-patientswith-head-and-neck-squamous-cell-carcinomas-a-10-year-single-center-experience-inchinese-population

\section{Hosted file}

Figure 2.docx available at https://authorea.com/users/323818/articles/452304-value-ofoesophagoscopy-and-bronchoscopy-in-diagnosis-of-synchronous-malignancies-in-patientswith-head-and-neck-squamous-cell-carcinomas-a-10-year-single-center-experience-inchinese-population

\section{Hosted file}


Table 1.docx available at https://authorea.com/users/323818/articles/452304-value-ofoesophagoscopy-and-bronchoscopy-in-diagnosis-of-synchronous-malignancies-in-patientswith-head-and-neck-squamous-cell-carcinomas-a-10-year-single-center-experience-inchinese-population

\section{Hosted file}

Table 2.docx available at https://authorea.com/users/323818/articles/452304-value-ofoesophagoscopy-and-bronchoscopy-in-diagnosis-of-synchronous-malignancies-in-patientswith-head-and-neck-squamous-cell-carcinomas-a-10-year-single-center-experience-inchinese-population 